13.1

\title{
Поверхностное соединение при адсорбции Ве на W(100): определение абсолютной концентрации и свойства
}

\author{
() Е.В. Рутьков, Е.Ю. Афранасьева, Н.Р. Галль \\ Физико-технический институт им. А.Ф. Иофффе РАН, Санкт-Петербург, Россия \\ E-mail: afanaseva@ms.ioffe.ru
}

Поступило в Редакцию 27 августа 2021 г.

В окончательной редакции 18 октября 2021 г.

Принято к публикации 19 октября 2021 г.

Показано, что адсорбция Ве на $\mathrm{W}(100)$ в области температур 900-1100К приводит к образованию стабильного адсорбционного состояния, при формировании которого значительно уменьшается энергия активации растворения атомов Ве и все вновь поступающие атомы растворяются в объеме подложки. Абсолютная концентрация атомов Ве в этом поверхностном соединении определена с использованием разработанного нами специального гетерного сверхвысоковакуумного источника Ве методом электронной оже-спектроскопии. Оказалось, что эта концентрация составляет $(1.0 \pm 0.1) \cdot 10^{15} \mathrm{~cm}^{-2}$ и соответствует стехиометрии WBe. При $T>1100 \mathrm{~K}$ пленка разрушается, и атомы Ве растворяются в объеме с энергией активации $\sim 3.5 \mathrm{eV}$.

Ключевые слова: адсорбция, поверхность, бериллий, вольфрам, твердый раствор.

DOI: 10.21883/PJTF.2022.03.51977.19005

Элементы первого и второго периодов с $s$ - и p-электронами являются наиболее распространенными примесями и технологически значимыми лигандами, определяющими свойства большинства переходных металлов и их сплавов. Адсорбция этих элементов на поверхности тугоплавких металлов позволяет прогнозировать основные свойства сплавов, так как именно внутренние поверхности (границы зерен) играют ключевую роль в физическом материаловедении [1-3]. Важнейший фактор - образование на поверхности специфических адсорбционных состояний, поверхностных соединений, которые на много порядков меняют скорости прохождения атомов через поверхностные диффузионные барьеры $[4,5]$.

Бериллий является одним из наиболее перспективных элементов для аэрокосмической отрасли и лигандов в металлургии; его сплавы широко применяются в приборостроении и оборонной промышленности $[6,7]$. С физической и химической точек зрения бериллий один из интереснейших элементов, сочетающих металлическую проводимость, малый атомный размер с очень высоким потенциалом ионизации $(\sim 9 \mathrm{eV})$, характерным для атомов неметаллов [8]. Особое значение имеет адсорбция бериллия на вольфраме, так как именно это сочетание широко используется как материал первой стенки в современных токамаках, в частности в ITER (International Thermonuclear Experimental Reactor).

В литературе имеется ряд работ, посвященных адсорбционной системе $\mathrm{Be}-\mathrm{W}$ [9-14]. В экспериментальных работах $[9,12,14]$ методом рентгеновской фотоэлектронной спектроскопии исследуются термостабильность нанометровых бериллиевых пленок и образование объемных соединений. Общим недостатком этих работ является отсутствие данных об абсолютных концентрациях нанесенного бериллия, что связано прежде всего с технологическими сложностями его стабильного напыления в сверхвысоком вакууме. Настоящая работа посвящена определению абсолютной концентрации атомов бериллия на W(100).

Опыты проводились в сверхвысоковакуумном электронном оже-спектрометре высокого разрешения $(\Delta E / E \sim 0.1 \%)[15]$. Образцами служили тонкие ленты из вольфрама размером $50 \times 1 \times 0.02 \mathrm{~mm}$ с работой выхода $е \varphi=4.65 \mathrm{eV}$, что соответствует грани (100), причем поверхность однородна по работе выхода. По данным дифракционной рентгеновской спектроскопии степень выхода грани составляла 99.5\%.

Бериллий напылялся из специально разработанного источника, основанного на растворении атомов Ве в иридии и его последующей термодесорбции. Конструкция источника будет описана в отдельной публикации.

На рис. 1 показано изменение интенсивности ожесигналов $\mathrm{Be}(E=104 \mathrm{eV})$ и $\mathrm{W}(E=163 \mathrm{eV})$ при напылении Ве постоянным потоком на $\mathrm{W}(100)$ при $T=1100 \mathrm{~K}$. Видно, что рост оже-сигнала Ве почти линейный, а при $t \geqslant 70 \mathrm{~s}$ интенсивность сигнала достигает насыщения („полочка“). Аналогичные кривые с выходом на „Полочку“ наблюдались и при изменении температуры вольфрамовой ленты в диапазоне 900-1100 K.

Можно ожидать, что аналогично другим адсорбатам, например $\mathrm{Si}, \mathrm{P}, \mathrm{S}, \mathrm{C}, \mathrm{O}$, образующим с вольфрамом поверхностные соединения (ПС) стехиометрии $\mathrm{WX}(X-$ атом адсорбата) [4], бериллий также образует ПС с вольфрамом. Линейный рост концентрации атомов Ве при напылении, независимость формы кривой от $T$, а также слабая экранировка оже-сигнала W (в 1.8 раза) указывают на то, что все напыленные атомы Ве остаются на поверхности, как это было и с атомами $\mathrm{Si}, \mathrm{P}$ и $\mathrm{S}$, 


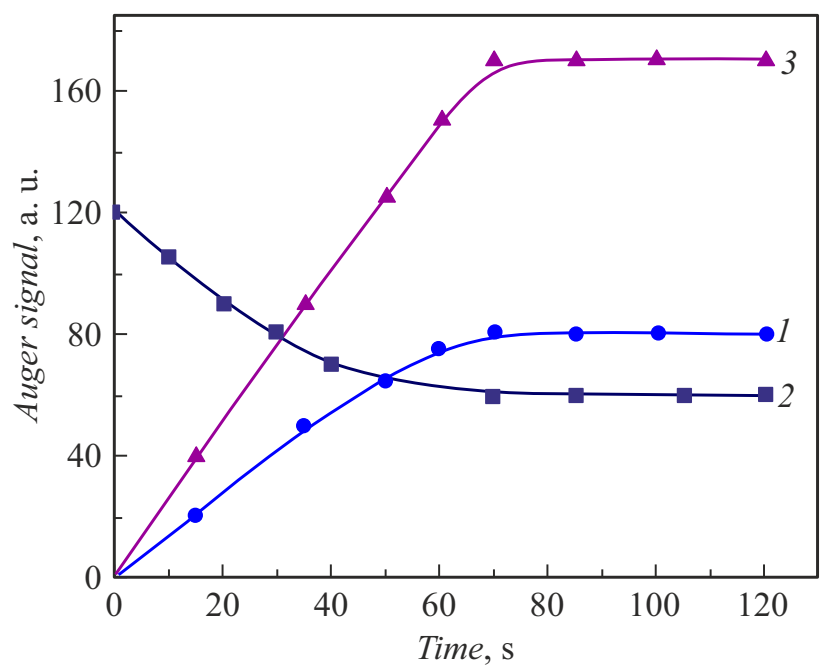

Рис. 1. Изменение интенсивности оже-сигналов бериллия (1), вольфрама (2) и фосфора (3) в зависимости от времени при адсорбции бериллия или фосфора в соединении $\mathrm{PCl}_{3}$ на $\mathrm{W}(100)$ при $T=1100 \mathrm{~K}$.

т. е. он действительно формирует стабильное адсорбционное состояние, аналогичное ПС.

Для определения абсолютной концентрации напыленного Ве в ПС мы провели сравнение интенсивности его оже-сигнала с оже-сигналами других адсорбатов, атомы которых также располагаются строго на поверхности с известными, ранее определенными концентрациями. Для этого были использованы атомы Р и $\mathrm{Si}$.

Динамика адсорбции фосфора на той же вольфрамовой ленте при $T=1100 \mathrm{~K}$ представлена на рис. 1 (кривая 3). Для этого использовались молекулы $\mathrm{PCl}_{3}$ : при $T>1000 \mathrm{~K}$ молекулы разваливаются, хлор в виде $\mathrm{Cl}_{2}$ десорбируется, а фосфор остается на поверхности вольфрама, образуя ПС стехиометрии WP, где $N_{W}=N_{P}=1 \cdot 10^{15}$ atom $/ \mathrm{cm}^{2}$ [16]. Из атласа интенсивностей оже-спектров химических элементов для случая электронной оже-спектроскопии (ЭОС) [17] следует, что фосфор примерно в 2.1 раза лучше „чувствуется“ методом ЭОС, чем бериллий. Из рис. 1 (кривые 1 и 3 ) видно, что интенсивность оже-сигнала фосфора $(E=120 \mathrm{eV})$ в 2.05 раза выше, чем у бериллия $(E=104 \mathrm{eV})$. Близость оже-пиков по энергии и использование единой подложки позволяет проводить прямое сравнение их интенсивностей, не учитывая вклад обратнорассеянных электронов. Поэтому можно, видимо, утверждать, что концентрация атомов $\mathrm{P}$ и $\mathrm{Be}$ на $\mathrm{W}(100)$ в поверхностных соединениях одинакова, а следовательно, бериллий на вольфраме образует ПС типа WBe, где $N_{\mathrm{Be}}=(1.0 \pm 0.1) \cdot 10^{15}$ atom $/ \mathrm{cm}^{2}$.

Аналогичный опыт был повторен с использованием в качестве калибровочного стандарта ПС кремния на $\mathrm{W}$ [5]. Из прямого сравнения интенсивностей ожесигналов $I_{\mathrm{Si}}(E=92 \mathrm{eV})$ и $I_{\mathrm{Be}}$ была получена величина
$I_{\mathrm{Si}} / I_{\mathrm{Be}}=3.4 \pm 0.2$, что хорошо согласуется с величиной из атласа спектров элементов $I_{\mathrm{Si}} / I_{\mathrm{Be}}=3.5$ [17].

Таким образом, на $\mathrm{W}(100)$ бериллий при адсорбции в области температур 900-1100 К образует ПС стехиометрии WBe.

Рассмотрим термостабильность ПС бериллия с вольфрамом. На рис. 2 показано изменение интенсивности оже-сигнала $\mathrm{Be}$ и W при отжиге ПC WBe. Видно, что при $T>1100 \mathrm{~K}$ соединение разрушается, и при $T=1400 \mathrm{~K}$ поверхность полностью свободна от бериллия, при этом интенсивность оже-сигнала W восстанавливается до прежней величины (кривая 2 на рис. 2). Уход Ве с поверхности может быть связан как с десорбцией бериллия, так и с его растворением в объеме вольфрама. Чтобы разрешить этот вопрос, применяли дополнительную вольфрамовую ленту, расположенную на расстоянии $\sim 20 \mathrm{~mm}$ под углом $45^{\circ}$ к рабочей ленте. На дополнительной ленте при $T=1000 \mathrm{~K}$ формировали ПC WBe, далее образец нагревали. Ожидаемые продукты десорбции наблюдались на рабочей ленте методом ЭОС только при нагреве дополнительной ленты выше $2400 \mathrm{~K}$. Поэтому уход атомов бериллия с поверхности при $T=1400 \mathrm{~K}$ связан только с растворением Ве в объеме подложки.

На рис. 3 показана кинетика изменения интенсивности оже-сигнала Ве и $\mathrm{W}$ при растворении бериллия в вольфраме при $T=1300 \mathrm{~K}$. Если воспользоваться известным выражением для времен жизни $\tau$ адатомов на поверхности $\tau=\tau_{0} \exp (E / k T)$ [18] и положить $\tau_{0}=10^{-13} \mathrm{~s}$, то можно оценить величину энергии активации перехода атомов Ве с поверхности в объем вольфрама: $E=3.5-3.7 \mathrm{eV}$, при этом наблюдается слабая концентрационная зависимость $E=f\left(N_{\mathrm{Be}}\right)$. Эта величина $E$ хорошо согласуется с рассчитанной теоретически для грани (100) W в работе [13].

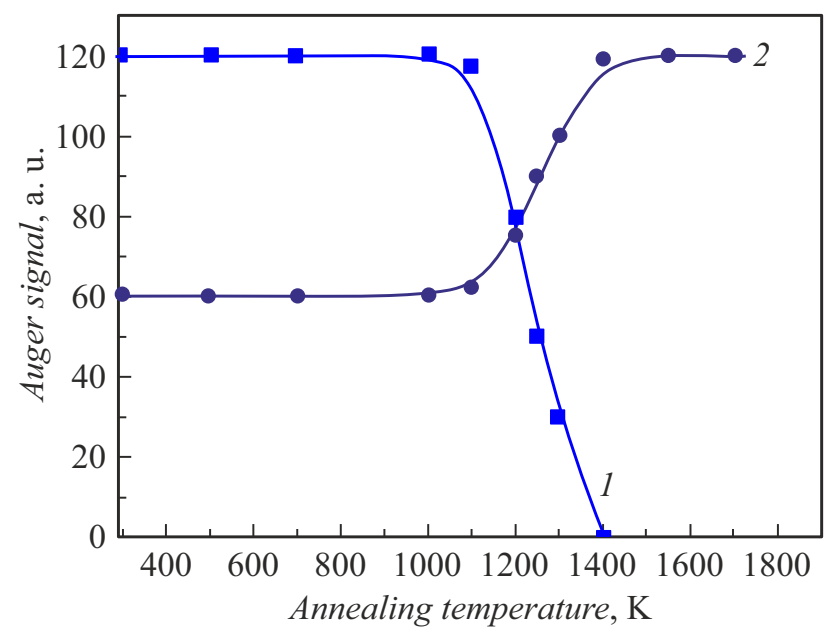

Рис. 2. Изменение интенсивности оже-сигналов бериллия (1) и вольфрама (2) при отжиге поверхностного соединения WBe при различных температурах. Время отжига в каждой температурной точке $30 \mathrm{~s}$. 


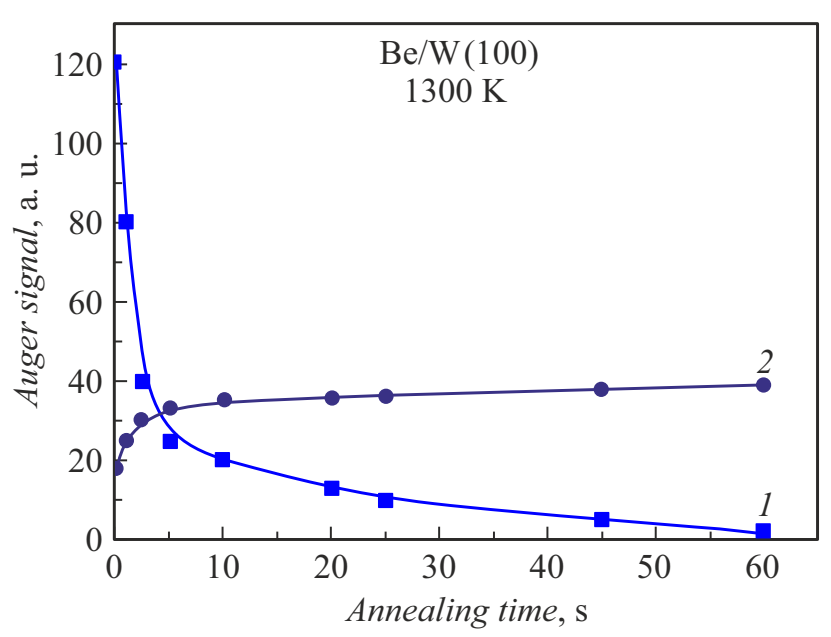

Рис. 3. Изменение интенсивности оже-сигналов бериллия (1) и вольфрама (2) в зависимости от времени при отжиге поверхностного соединения WBe при $T=1300 \mathrm{~K}$.

Как показано в наших работах [4,5], образование ПС связано с тем фактом, что на поверхности металлов $(\mathrm{W})$ имеются центры с предпочтительной по величине химической связью с адсорбатом, которые и занимаются в первую очередь. Только после их заполнения поступающие атомы начинают либо растворяться в объеме металла $(\mathrm{Si}, \mathrm{C})$, либо десорбироваться $(\mathrm{S}, \mathrm{P}, \mathrm{O})[4,5]$, причем энергия активации соответствующего процесса меняется резко, вызывая изменения скорости соответствующего процесса в сотни тысяч и миллионы раз. В случае Ве, видимо, наблюдается схожая ситуация. Опыты показали, что если адсорбировать атомы $\mathrm{Si}$ при $T=1000 \mathrm{~K}$ на ПС WBe (все сильносвязанные центры заняты атомами $\mathrm{Be}$ ), то кремний не накапливается на поверхности и сразу переходит в растворенное состояние. Отметим, что поверхностные соединения вступают в химические реакции с атомами других веществ [19]. В нашей работе, например, напыление атомов Ве в количестве $N_{\mathrm{Be}}=1 \cdot 10^{15}$ atom $/ \mathrm{cm}^{2}$ на ПС WSi при $T=1000 \mathrm{~K}$ приводило к полному замещению атомов $\mathrm{Si}$, которые растворяются в объеме вольфрама.

Таким образом, показано, что напыление атомов Ве на поверхность $\mathrm{W}(100)$ приводит к образованию специфического адсорбционного состояния - поверхностного соединения, аналогичного тем, которые образовывали на $\mathrm{W}(100)$ атомы $\mathrm{Si}, \mathrm{S}, \mathrm{P}, \mathrm{C}$ и О. С помощью специально разработанного нами гетерного сверхвысоковакуумного источника и метода электронной оже-спектроскопии определена абсолютная концентрация Ве в этом состоянии, что позволило воссоздать физическую картину протекающих процессов и использовать грань $(100) \mathrm{W}$ в качестве „калибровочной“ поверхности для многих атомов, в том числе и для Ве. Можно предположить, что аналогичные ПС с участием Ве будут образовываться и на других подложках, особенно стабильных и с выраженным атомным рельефом.

\section{Конфликт интересов}

Авторы заявляют, что у них нет конфликта интересов.

\section{Список литературы}

[1] Д. Мак Лин, Гранищы зерен в металлах (Металлургиздат, M., 1960). [D. McLean, Grain boundaries in metals (Clarendon Press, Oxford, 1957).].

[2] M. Guttmann, D. McLean, in Interfacial segregation, ed. by W.C. Johnson, J.M. Blakely (American Society for Metals, Metal Park, Ohio, 1979), p. 261-347.

[3] A. Atkinson, J. de Phys. Coll., 46, C4-379 (1985). DOI: $10.1051 /$ jphyscol:1985441

[4] Н.Р. Галль, Е.В. Рутьков, А.Я. Тонтегоде, Рос. хим. журн., 47 (2), 13 (2003).

[5] В.Н. Агеев, Е.Ю. Афанасьева, Н.Р. Галль, Е.В. Рутьков, С.Н. Михайлов, А.Я. Тонтегоде, Письма в ЖТФ, 12 (9), 565 (1986).

[6] В. Эспе, Технология электровакуумных материалов (Госэнергоиздат, М.-Л., 1962), т. 1.

[7] B.N. Kolbasov, V.I. Khripunov, A.Yu. Biryukov, Fusion Eng. Des., 109-111 (Pt A), 480 (2016).

DOI: $10.1016 /$ j.fusengdes.2016.02.073

[8] Г.Ф. Силина, Ю.И. Зарембо, Л.Э. Бертина, Бериллий, химическая технология и металлургия (Атомиздат, М., 1960).

[9] A. Wiltner, Ch. Linsmeier, J. Nucl. Mater., 337-339, 951 (2005). DOI: 10.1016/j.jnucmat.2004.08.021

[10] L. Chen, I. Sukuba, M. Probst, A. Kaiser, Nucl. Mater. Energy, 16, 149 (2018). DOI: 10.1016/j.nme.2018.06.021

[11] L. Chen, D. Süß, I. Sukuba, M. Schauperl, M. Probst, Th. Maihom, A. Kaiser, Nucl. Mater. Energy, 22, 100731 (2020). DOI: 10.1016/j.nme.2020.100731

[12] A. Wiltner, C. Linsmeier, New J. Phys., 8, 181 (2006). DOI: $10.1088 / 1367-2630 / 8 / 9 / 181$

[13] A. Allouche, A. Wiltner, C. Linsmeier, J. Phys.: Condens. Matter, 21, 355011 (2009).

DOI: $10.1088 / 0953-8984 / 21 / 35 / 355011$

[14] Ch. Linsmeier, K. Ertl, J. Roth, A. Wiltner, K. Schmid, F. Kost, S.R. Bhattacharyya, M. Baldwin, R.P. Doerner, J. Nucl. Mater., 363-365, 1129 (2007). DOI: 10.1016/j.jnucmat.2007.01.224

[15] В.Н. Агеев, Е.В. Рутьков, А.Я. Тонтегоде, Н.А. Холин, ФТТ, 23 (8), 2248 (1981).

[16] Н.Р. Галль, Е.В. Рутьков, А.Я. Тонтегоде, Письма в ЖТФ, 26 (12), 31 (2000). [N.R. Gall, E.V. Rut'kov, A.Ya. Tontegode, Tech. Phys. Lett., 26 (6), 510 (2000). DOI: $10.1134 / 1.1262894]$.

[17] Handbook of Auger electron spectroscopy (Physical Electronics Industries, Edina, 1976).

[18] Э.Я. Зандберг, Н.И. Ионов, Поверхностная ионизация (Наука, M., 1969). [Е.Ya. Zandberg, N.I. Ionov, Surface ionization (Israel Program for Scientific Translation, Jerusalem, 1971).

[19] Н.Р. Галль, Е.В. Рутьков, А.Я. Тонтегоде, Письма в ЖТФ, 30 (19), 72 (2004). [N.R. Gall, E.V. Rut'kov, A.Ya. Tontegode, Tech. Phys. Lett., 30 (10), 832 (2004).

DOI: $10.1134 / 1.1813724]$. 